
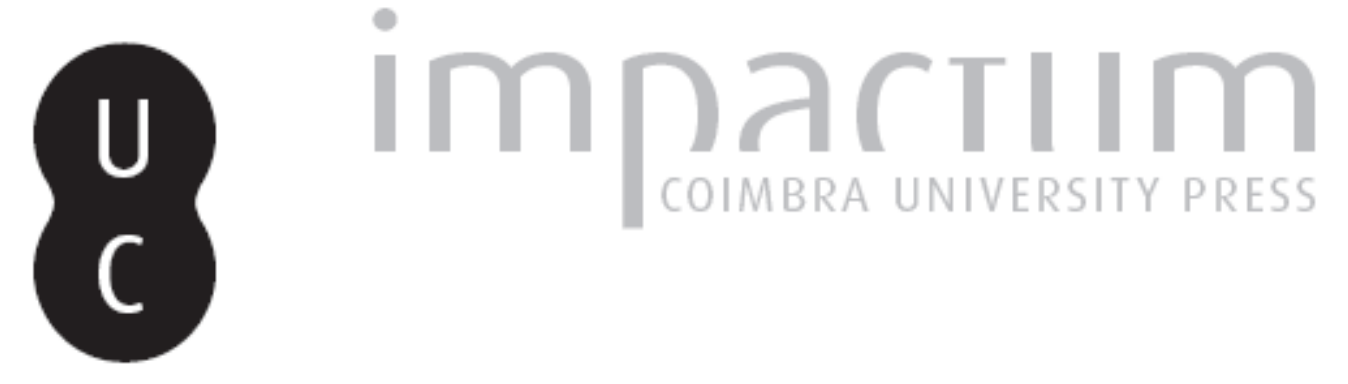

\title{
Tipo de circulação atmosférica que suscita risco de agudização de asma
}

\author{
Autor(es): $\quad$ Talaia, Mário; Pina, Denise
}

Publicado por: $\begin{aligned} & \text { Associação Portuguesa de Riscos, Prevenção e Segurança; Imprensa } \\ & \text { da Universidade de Coimbra }\end{aligned}$

URL

persistente:

URI:http://hdl.handle.net/10316.2/39793

DOI:

DOI:https://doi.org/10.14195/1647-7723_23_15

Accessed : $\quad$ 26-Apr-2023 16:12:29

A navegação consulta e descarregamento dos títulos inseridos nas Bibliotecas Digitais UC Digitalis, UC Pombalina e UC Impactum, pressupõem a aceitação plena e sem reservas dos Termos e Condições de Uso destas Bibliotecas Digitais, disponíveis em https://digitalis.uc.pt/pt-pt/termos.

Conforme exposto nos referidos Termos e Condições de Uso, o descarregamento de títulos de acesso restrito requer uma licença válida de autorização devendo o utilizador aceder ao(s) documento(s) a partir de um endereço de IP da instituição detentora da supramencionada licença.

Ao utilizador é apenas permitido o descarregamento para uso pessoal, pelo que o emprego do(s) título(s) descarregado(s) para outro fim, designadamente comercial, carece de autorização do respetivo autor ou editor da obra.

Na medida em que todas as obras da UC Digitalis se encontram protegidas pelo Código do Direito de Autor e Direitos Conexos e demais legislação aplicável, toda a cópia, parcial ou total, deste documento, nos casos em que é legalmente admitida, deverá conter ou fazer-se acompanhar por este aviso.

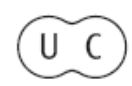




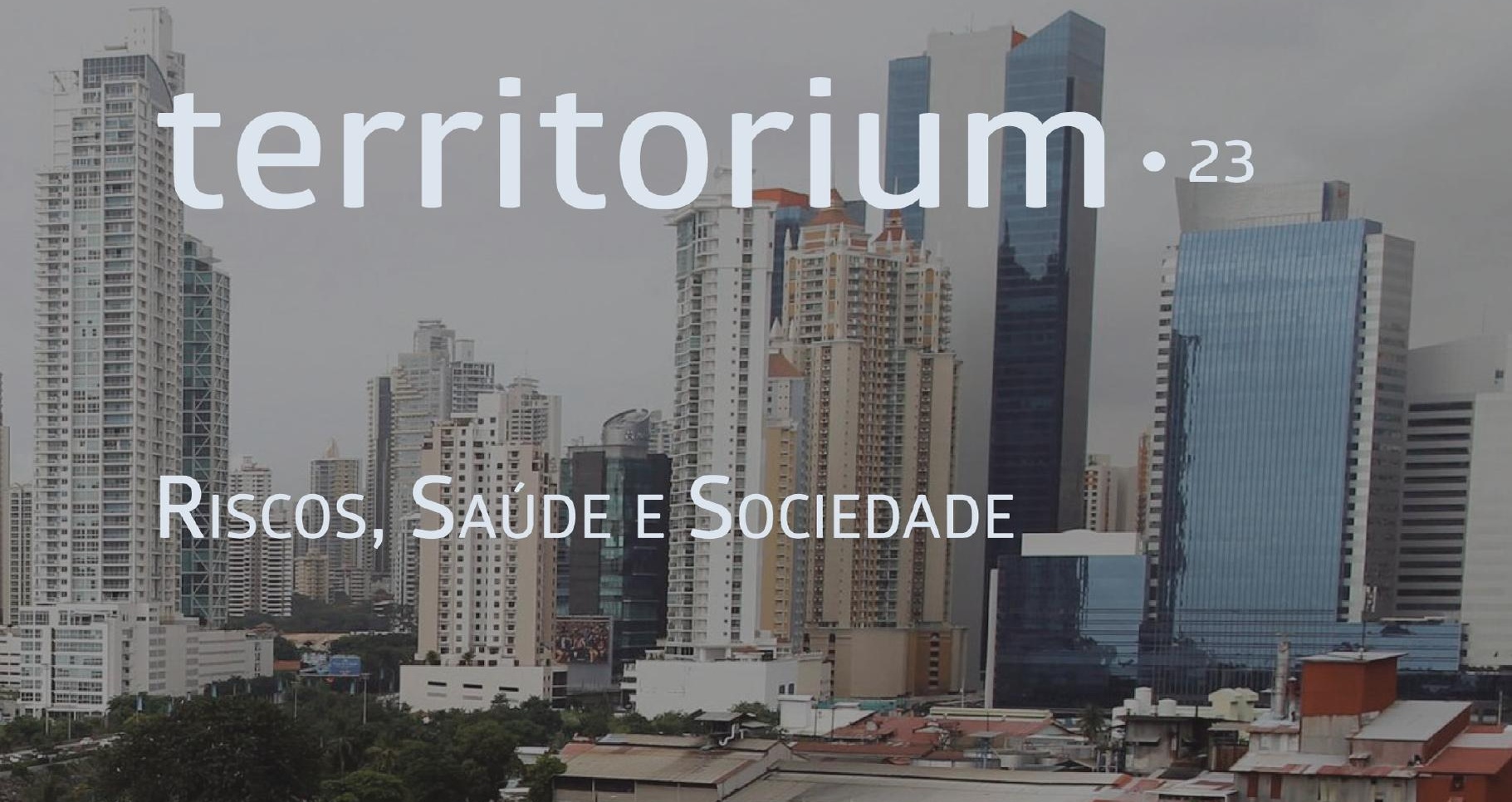

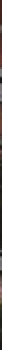

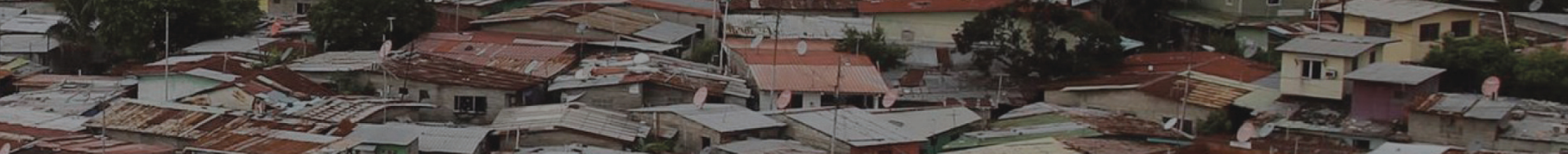

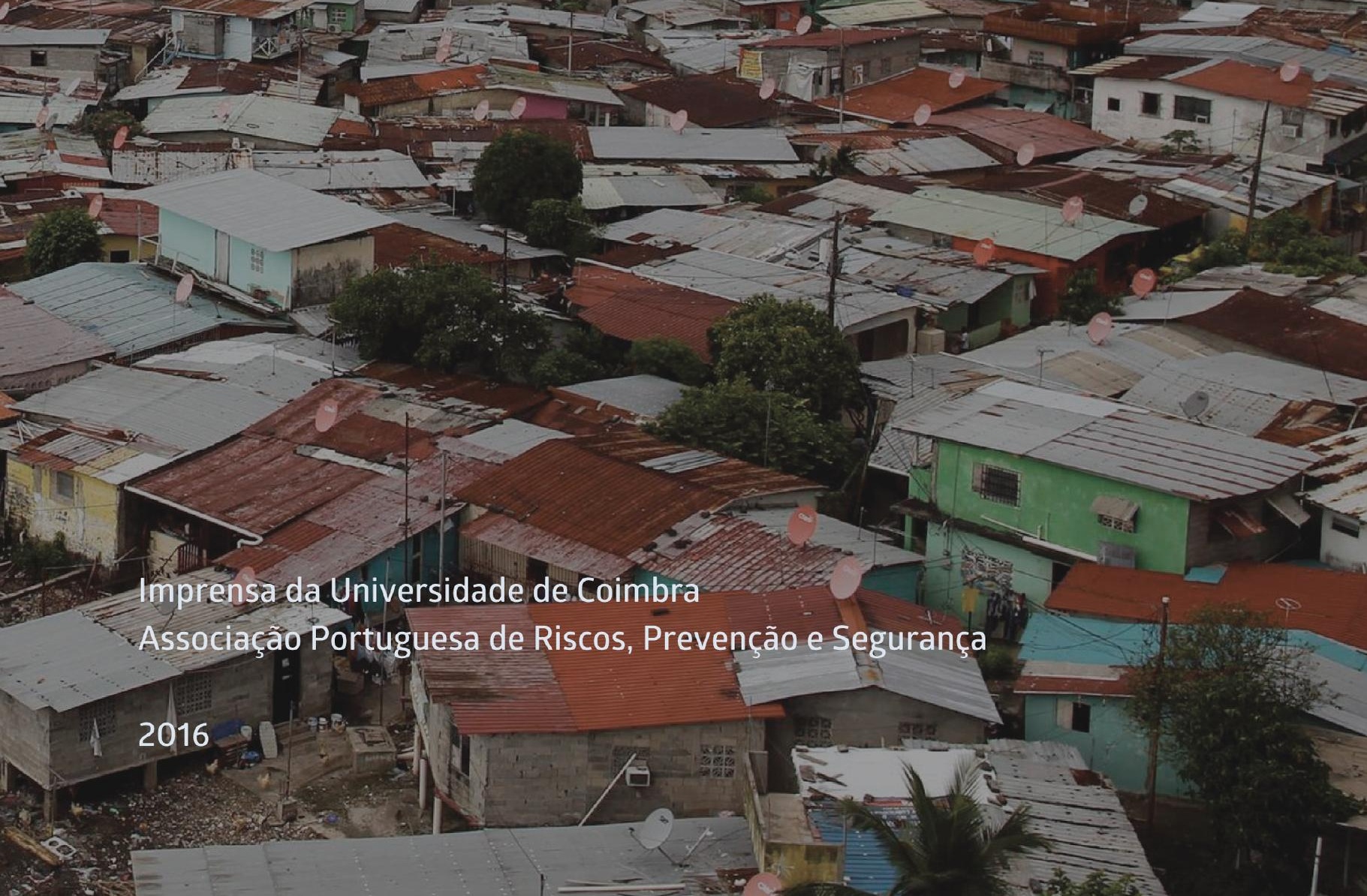




\section{TIPO DE CIRCULAÇÃO ATMOSFÉRICA QUE SUSCITA RISCO DE AGUDIZAÇÃO DE ASMA*}

CIRCULATION WEATHER TYPES AND THEIR INFLUENCE ON THE RISK OF ASTHMA ATTACKS

Mário Talaia

Departamento de Física da Universidade de Aveiro mart@ua.pt

Denise Pina

Departamento de Física da Universidade de Aveiro denisesp@ua.pt

\section{RESUMO}

Neste trabalho é estudado o risco de agravamento de ataques asmáticos. Foram usados dados de uma estação meteorológica e acorrências hospitalares, durante um ano. Investigaram-se os tipos de circulação atmosférica que estão associados ao risco de agravamento da doença. Os resultados obtidos mostraram que um tipo de circulação atmosférico SW com frente fria é um risco de agravamento da doença e estratégias de intervenção devem ser adoptadas.

Palavras-chave: Tipos de circulação atmosférica, asma, frente fria, acorrências hospitalares.

\section{ABSTRACT}

This paper examines the risk of asthma attacks. It was used data from a weather station and hospital emergencies for a year. The types of atmospheric circulation that are associated with the risk of disease were studied. The results show that a type of atmospheric circulation SW with cold front is a risk of disease and intervention strategies should be adopted.

Keywords: Circulation weather types, asthma, cold front, hospital emergencies.

\section{Introdução}

Desde os tempos mais remotos que as doenças respiratórias influenciam a qualidade de vida das pessoas. Na sua obra, S. W. Tromp (1980) mostra que o ser humano sempre teve necessidade de compreender a influência do risco do tempo atmosférico, do clima, da poluição na agudização de doenças de índole respiratória.

No seu texto "Ares, águas, lugares" Hipócrates (460 AC a $370 \mathrm{AC}$ ) considerado o "pai da medicina" já discutia os fatores ambientais ligados à doença, defendendo um conceito ecológico de saúde-enfermidade. Essas observações não se limitavam ao paciente em si, mas a seu ambiente (H. F. Cairus e Jr. Ribeiro, 2006).

$\mathrm{Na}$ segunda metade do século XIX, em especial na Europa, houve grande desenvolvimento dos estudos que relacionavam algumas das principais doenças com as condições meteorológicas e climáticas regionais, que nalguns casos levaram à expansão da utilização das estâncias termais e também dos sanatórios; em Portugal deve referir-se, nesta época, o trabalho do médico
Dr. Joaquim Guilherme Gomes Coelho, que ficou mais conhecido pela sua actividade literária com o nome de Júlio Dinis, que defendeu a tese 'Da importância dos estudos meteorológicos para a medicina e, especialmente, das suas aplicações ao ramo cirúrgico' no seu doutoramento em Medicina, em 1861, na Universidade do Porto (R. A. C. Carvalho, 1999; Júlio Diniz, 2005).

Fica aqui expressa a homenagem a Renato de Carvalho ilustre meteorologista de Portugal, que em 1999 escreveu um artigo com o título "A influência das condições meteorológicas e do clima sobre doenças humanas". Neste artigo Renato de Carvalho escreve que "os efeitos da influência das condições meteorológicas e do clima foram largamente estudados ao longo dos tempos, sendo bem conhecidas as aplicações que eram feitas durante o Império Romano nas estâncias termais e mais tarde nos sanatórios, quer localizados em áreas com climas secos, em regra frios, que se localizavam em regiões montanhosas, quer localizados à beira-mar, que tanta divulgação tiveram desde a segunda metade do século XIX e até meados do século XX. Em 1964 a Organização

* O texto desta nota corresponde a uma comunicação apresentada no III Congresso Internacional, I Simpósio Ibero-Americano e VIII Encontro Nacional de Riscos, tendo sido submetido em 04-02-2015, sujeita a revisão por pares a 18-06-2015 e aceite para publicação em 31-03-2016.

Esta nota é parte integrante da Revista Territorium, $n .{ }^{\circ} 23,2016,{ }^{\circ}$ RIscos, ISSN: 0872-8941. 
Meteorológica Mundial promoveu a publicação contida na nota técnica $n^{\circ} 65$ intitulada 'Survey of human biometeorology', onde se apresentava a sinopse dos 'Efeitos da influência do estado do tempo e do clima sobre as doenças'. Nesta sinopse pode reconhecer-se que as crises de doenças do aparelho respiratório (asma brônquica) estão, em regra, associadas ao tempo frio e húmido, com nevoeiros, ar poluído e quedas bruscas da temperatura do ar; também com tempo quente opressivo (antes de trovoadas e depois do efeito de Fõhn). Com efeito, é reconhecido que as variações térmicas acentuadas são determinantes no agravamento de numerosas doenças. Assim as descidas acentuadas da temperatura do ar (vagas de frio) provocam o agravamento de doenças, de que se destacam as respiratórias, incluindo a asma. No entanto, também as subidas acentuadas da temperatura do ar com a duração de alguns dias (vagas de calor) podem provocar situações críticas na saúde pública" (R. A. C. Carvalho, 1999).

A obra Thermal Comfort de P. Fanger (1972) veio confirmar a importância do estudo do ambiente térmico e enfatizar o carácter multi e interdisciplinar desta área de estudo. A publicação de legislação relacionada com o ambiente térmico, pela ISO (International Organization for Standartization) e pela ASHRAE (American Society for Heating, Refrigeration and Air-Conditioning Engineers) veio reforçar a importância do estudo desta temática e a obrigatoriedade de a legislar de forma a salvaguardar a saúde do ser humano.

Hoje em dia em recintos fechados é possível controlar a temperatura e a humidade relativa para se ter um maior conforto térmico ou seja uma melhor qualidade de vida. A procura de uma boa qualidade de vida é um direito de qualquer cidadão. A legislação portuguesa referente a ambientes térmicos confortáveis e de qualidade é ainda muito vaga. A portaria $n .^{\circ} 702 / 80$ de 22 de Setembro refere alguns cuidados a ter com a temperatura $e$ humidade relativa do ar para espaços fechados. Com a aprovação em 2006 da legislação especifica que Regulamenta os Sistemas Energéticos de Climatização e a Qualidade do Ar Interior nos Edifícios (Decreto-Lei n. $\left.{ }^{\circ} 79 / 2006\right)$ verificou-se uma melhoria na climatização e na qualidade do ar interior. No entanto, há muito a fazer e muito a investigar nesta área, uma vez que a informação existente é escassa e a sua aplicação nem sempre é linear.

Espera-se que nos próximos 50 anos, os combustíveis fosseis e a biomassa continuarão a ser certamente as principais fontes de energia do mundo e é bem conhecido que a exposição aos poluentes, resultantes da produção de energia através destes processos, pode ter como consequência sérios efeitos a nível da saúde, especialmente se ocorrerem episódios severos de poluição (R. K. Bush and Prochnau, 2004; C. P. Weisel et al. (2002), S. Boutin-Forzado et al. (2004) e M. Talaia et al. (2007) mostraram que existe uma relação estatisticamente significativa entre as acorrências de asma (ou seja as emergências diárias hospitalares) e os valores da concentração de alguns poluentes.

De acordo com J. L. Plácido (2004) a asma é, actualmente, considerada em todo o mundo como um importante problema de saúde publica, afectando milhões de pessoas de todas as faixas etárias, podendo nalguns casos ser potencialmente fatal, com um enorme impacto na qualidade de vida do doente e da sua família e com custos económicos importantes. Apesar de existirem muitos trabalhos científicos acerca da incidência, prevalência e mortalidade por asma, sempre existiram grandes dificuldades metodológicas na sua realização e comparação pelo facto da asma não ser fácil de definir e caracterizar em termos epidemiológicos.

A asma é uma doença inflamatória. Nos indivíduos susceptíveis, esta inflamação causa sintomas que estão habitualmente associados a uma difusa, mas variável, obstrução dos fluxos aéreos, que é, muitas vezes, reversível espontaneamente ou com tratamento. As agudizações de asma manifestam-se por dificuldade respiratória, devido ao estreitamento generalizado das vias aéreas pulmonares, por constrição das fibras musculares dos brônquios de pequeno calibre. A passagem do ar pelas vias aéreas pode ser prejudicada por espasmos da musculatura dos brônquios e bronquíolos, por edema (aumento de fluido) nas membranas mucosas que o revestem, e pelo aumento da produção de muco. Essas alterações podem originar deficiências de oxigenação, provocando a crise asmática. A asma é uma doença complexa. Na realidade, os asmáticos não compreendem o que se passa com eles, e isso pode dar origem a ansiedade, o que parece agravar a doença ( $M$. C. Almeida, 2013).

As causas da agudização da asma parecem depender de inúmeros factores, nomeadamente o estado emocional do paciente, o tipo de residência, o tipo de trabalho e o tipo de clima. Para um asmático, podem existir diversas substâncias que produzem uma hipersensibilidade nos brônquios. Quando os brônquios se tornam sensíveis, factores como mudanças de temperatura, variação na humidade do ar, choques emocionais ou até exercícios físicos (excesso de esforços) podem precipitar a agudização (L. S. Girsh et al., 1967; M. C. Piccolo et al., 1988 e S. Suzuki et al., 1988).

A região de Aveiro, normalmente regista humidades relativas do ar altas durante todo o ano, valores que são condicionados pela existência de uma evaporação activa. Na prática, poder-se-á afirmar que é uma região que se pode tornar um excelente lugar de proliferação de ácaros. 0 habitat dos ácaros depende da temperatura do ar e da humidade relativa do ar. 
E. H. Derrick (1965) mostrou que uma massa de ar associada a uma temperatura do ar relativamente baixa (uma atmosfera fria) pode irritar a mucosa respiratória e suscitar agudização de asma. W. H. Van (2004) refere que a asma não tem uma causa muito clara mas que esta doença, em pessoas que são sensíveis aos ácaros, poderá ser causada por uma recorrente inalação deste agente alergénio. U. Gehring et al. (2001) concluíram que a exposição aos ácaros acima de determinadas concentrações aumenta o risco de ocorrência de uma respiração difícil e ruidosa e que a presença de pelos de animais, nomeadamente de gato, está associada à tosse nocturna.

A OMS refere que uma atmosfera fria é factor desencadeante da agudização de asma. J. Sousa e Talaia (2005) mostraram existir uma correlação significativa da gripe na agudização da asma, na presença de uma atmosfera fria. Interessante também é o estudo de P. F. Jamasson et al. (1997) que afirmaram ser evidente a relação entre certas massas de ar com o aumento das admissões hospitalares por agudizações de asma.

A SPAIC (Sociedade Portuguesa de Alergologia e Imunologia Clínica) refere no seu Manual Educacional do Doente que "[...] as mudanças súbitas das condições meteorológicas podem resultar em sintomas no doente asmático". Não são pormenorizados os factores nem dadas sugestões de prevenção. Também o documento nas Medidas de controlo ambiental, refere apenas "reduzir os factores desencadeantes".

M. Talaia e Sousa (2004) mostraram que a qualidade do ar é factor determinante nas agudizações da asma com acorrências hospitalares.

Segundo GOLD Report (2015) a asma pode ser um fator de risco para o desenvolvimento de DPOC (Doença Pulmonar Obstrutiva Crónica), embora a evidência não seja conclusiva. Alguns pacientes com DPOC têm características compatíveis com asma, e o aumento da falta de ar está associado às temperaturas baixas. C. Almeida et al. (2012) mostraram que a perda de FEV1 ou VEM1 (Volume Expiratório Máximo no $1^{\circ}$ segundo) é um indicador de DVO (Doença Ventilatória Obstrutiva) e o valor do Índice de Tiffeneau pode não ser suficiente para o diagnóstico da doença.

A DPOC é uma patologia crónica, de evolução lenta e progressiva, com grande impacto sobre a função respiratória, que pode estar sujeita a períodos de agudização e complicações graves. A DPOC pode ser diagnosticada através dos resultados obtidos usando uma espirometria (F. L. C. Lundgren et al., 2006). Em Portugal a doença afecta entre 500 a 600 mil pessoas e no mundo ocidental constitui a $4^{\text {a }}$ causa de morte nos indivíduos do sexo masculino na idade média de vida, prevendo a Organização Mundial de Saúde (OMS) que em 2020 venha a ocupar a $1^{\text {a }}$ causa de morte relacionada com problemas respiratórios (C .J. Murray e A. D. Lopes, 1997; T. Oga et al., 2003).

Com base nos pressupostos anteriormente referidos parece-nos oportuna esta investigação para um ambiente com características de "frio". Espera-se que o estudo possa contribuir para um melhor conhecimento do tipo de circulação atmosférica que agudiza os ataques asmáticos e que suscitam a acorrência hospitalar.

\section{Materiais e Métodos}

O estudo é considerado exploratório e "estudo de caso". Para o tratamento dos dados usou-se um método quantitativo e um método qualitativo.

Usaram-se dados referentes as acorrências (por acorrer a ajuda nos serviços de urgência hospitalar) de agudização de asma registadas nos serviços de urgência de hospital de Aveiro e dados registados na estação clássica de meteorologia da Universidade de Aveiro representativa da região afeta ao hospital, durante um ano.

Os dados registados (acorrências hospitalares e parâmetros meteorológicos) foram considerados em semanas do ano de modo a encontrar as semanas de maior risco.

Numa primeira abordagem os dados não foram normalizados. Esta análise permitiu conhecer as linhas de evolução do sexo masculino, do sexo feminino e total (independente do sexo) e compreender a influência de diferentes parâmetros meteorológicos no risco de acorrências hospitalares. Foram desenhadas linhas móveis com a probabilidade da acorrência ser de D4 (quatro dias antes ou depois da acorrência aos serviços de urgência hospitalar. Numa segunda abordagem e para uma análise de pormenor os dados foram normalizados (para a média das acorrências registadas diárias). Por conveniência as ordenadas dos gráficos comparativos de acorrência foram considerados no mesmo intervalo de valor em percentagem.

Para uma melhor compreensão da agudização da doença consideraram-se semanas do ano, pois a acorrência hospitalar pode acontecer durante um período de 7 dias. Nestas circunstancias e após a identificação das semanas de maior risco procurou-se saber que diferentes tipos de circulação atmosférica estavam associados (R. M. Trigo e C.C. Câmara, 2000). Um dos objetivos foi identificar o tipo de circulação atmosférica que suscitava o agravamento risco de agudização da doença e se estava associada à presença de frente fria.

\section{Resultados e Discussão}

A fig. 1 mostra, a título de exemplo, a frequência relativa das acorrências hospitalares para a asma, 
quando se considera a temperatura mínima do ar. A observação da figura mostra círculos a três cores (cor verde indica as frequências relativas para o sexo feminino; cor azul indica as frequências relativas para o sexo masculino e cor negra indica as frequências relativas totais independente do sexo). As linhas de cor correspondentes indicam a média móvel até quatro dias antes e depois da acorrência hospitalar, e mostram que as ocorrências dependem da temperatura mínima, com maior frequência entre cerca de $5^{\circ} \mathrm{C}$ a $15^{\circ} \mathrm{C}$. A evolução das linhas era a esperada pois os valores não estão normalizados. Também, as linhas sugerem não haver diferenças significativas nas frequências relativas quando se valoriza o sexo. A observação das linhas parece mostrar, ainda, que se regista um decréscimo das ocorrências quando a temperatura mínima do ar é superior a cerca de $15^{\circ} \mathrm{C}$. Esta afirmação não é totalmente correta, pois só com dados normalizados é possível esclarecer detalhadamente o risco de agudização de asma.

A fig. 2 mostra as acorrências para intervalos de temperatura mínima do ar. A observação do gráfico da figura sugere que as acorrências normalizadas são ligeiramente superiores quando a temperatura mínima do ar diminui.

A fig. 3 mostra como as acorrências são influenciadas para intervalos de humidade relativa do ar.

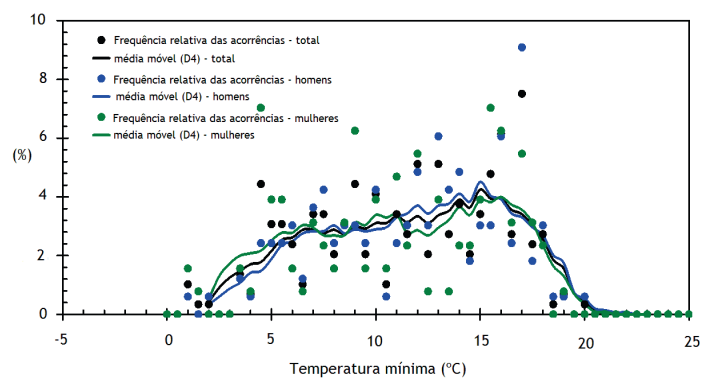

Fig. 1 - Frequência relativa das acorrências "versus" temperatura mínima do ar.

Fig. 1 - Relative frequencies of hospital emergencies admissions versus minimum air temperature.

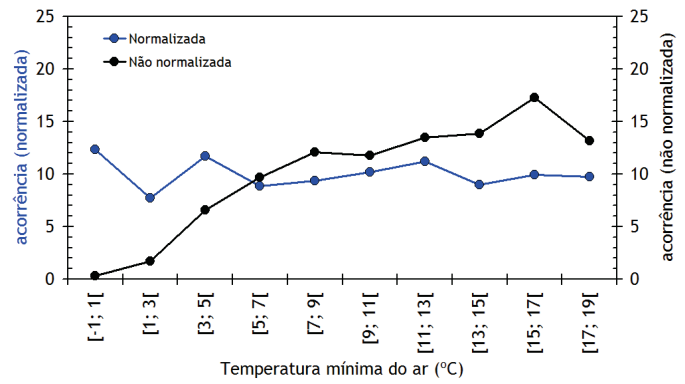

Fig. 2 - Acorrências “versus” temperatura mínima do ar.

Fig. 2 - Hospital emergencies admissions versus minimum air temperature.
A observação da fig. 3 mostra, como seria esperado, que as acorrências normalizadas aumentam quando a humidade relativa do ar aumenta. Deve-se salientar que estamos perante um cenário de temperatura mínima do ar. Os resultados obtidos mostram que há maior risco de acorrência para baixas temperaturas mínimas do ar. Esta situação está em concordância com a literatura (Derrick, 1965) quando afirma que frentes frias condicionam o risco de acorrência de surtos asmáticos.

$\mathrm{Na}$ prática e para o asmático, as baixas temperaturas mínimas do ar funcionam como constrição e por esta razão favorecem a dificuldade no ato de respirar o que suscita o risco de acorrência hospitalar. Nestes casos o muco ou o excesso de muco funciona como tampão nas vias respiratórias.

Para investigar a influência de diferentes tipos de circulações atmosféricas que favorecem o aumento significativo do risco de agudização de asma construi-se o gráfico da fig. 4.

A observação da fig. 4 mostra a linha de evolução das acorrências em cada semana, ao longo do ano. Para o ano de estudo, a observação do gráfico mostra inequivocamente que a partir da semana 36 há um aumento significativo das acorrências. Esta situação está em concordância com os resultados e análise realizada por R. A. Carvalho (1999).

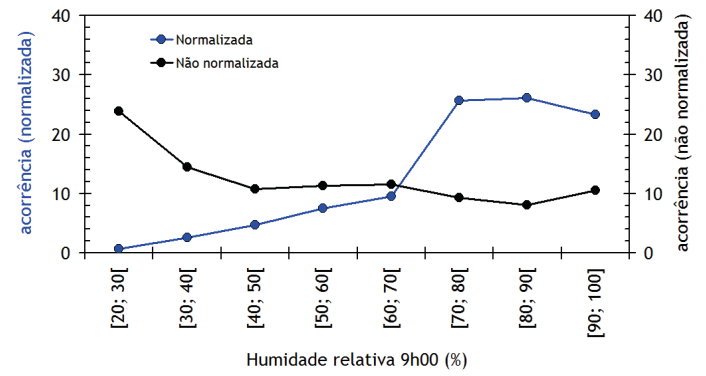

Fig. 3 - Acorrências "versus" humidade relativa do ar.

Fig. 3 - Hospital emergencies admissions versus relative humidity of air.

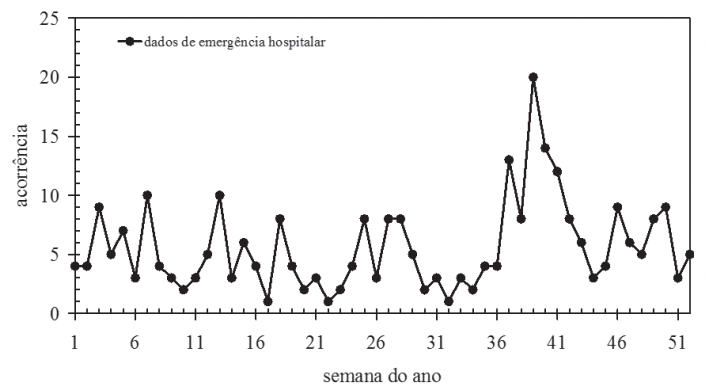

Fig. 4 - Acorrências "versus" semana do ano.

Fig. 4 - Hospital emergencies admissions versus week of the year. 
Fez-se então, uma análise, para as semanas de 36 a 42, recorrendo-se aos mapas do tempo diários retirados do site http://www.wetterzentrale.de/topkarten/tkfaxbraar.htm. Os resultados obtidos mostraram que há uma mudança do tipo de circulação quando se regista uma agudização da doença, ou seja, do tipo N/NE para SW (semana 37). A partir desta semana os pacientes da doença deviam ter tomado as suas estratégias de defesa, pois o risco de acorrência hospitalar aumenta. A análise das cartas do tempo diárias mostra que a circulação atmosférica do tipo SW e uma passagem de frente fria condiciona o risco de agravamento de acorrências.

A título de exemplo, a fig. 5 mostra mapas de tempo para a semana 40 (http://www.wetterzentrale.de/topkarten/ tkfaxbraar.htm).

A TABela I mostra para a semana 39 e 40 os Tipos de Circulação Atmosférica registados.

Como mostra a fig. 5, na semana 40 estão identificadas passagens de frentes frias que parecem suscitar o risco de agudização da doença e acorrências hospitalares. De fato, como já referido anteriormente, quando as temperaturas são baixas, há constrição e dificuldades no ato de respirar, favorecendo as ocorrências hospitalares. Os pacientes procuram ajuda hospitalar, para aliviar o gerado efeito tampão.
Há pacientes que são admitidos a uma recuperação em enfermaria. Este espaço deve ter um ar húmido com características termohigrométricas adequadas. Foram registados dados indoor e outdoor num dado momento, como exemplo. De acordo com o modelo de sensação térmica desenvolvido por $M$. Talaia e $\mathrm{H}$. Simões (2009) o valor do índice EsConTer mostrou ser de 1,02 para o interior da enfermaria e 0,01 para o exterior da enfermaria. Estas condições mostram que o interior da enfermaria apresentava um ambiente ligeiramente quente, o que indicia alguma dificuldade para a recuperação de doentes, pois o ar húmido não mostra sensação térmica confortável. Por outro lado, a temperatura do $\operatorname{ar}\left(26,3^{\circ} \mathrm{C}\right)$ e a humidade relativa do ar $(53,2 \%)$ da enfermaria indiciava uma necessidade de uma massa volúmica de vapor de água de $13,9 \mathrm{~g} / \mathrm{m}^{3}$. Este valor é superior ao que o ambiente outdoor registava de $10,9 \mathrm{~g} / \mathrm{m}^{3}$, muito próximo das condições de conforto térmico (temperaturas do ar de $20^{\circ} \mathrm{C}$ a $22^{\circ} \mathrm{C}$ e uma humidade relativa de $50 \%$ a $60 \%$, com cerca de $9,3 \mathrm{~g} /$ $\mathrm{m}^{3}$ ). Nestas circunstâncias, o valor da massa volúmica do ar húmido da enfermaria gera condições de "papel mata-borrão" o que pode suscitar risco de agudização da doença.

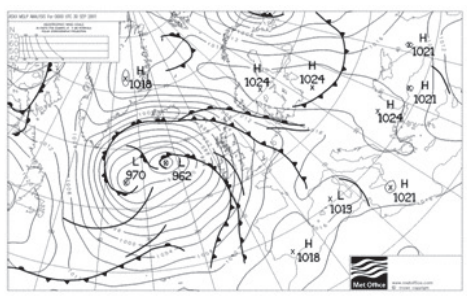

30 de Setembro

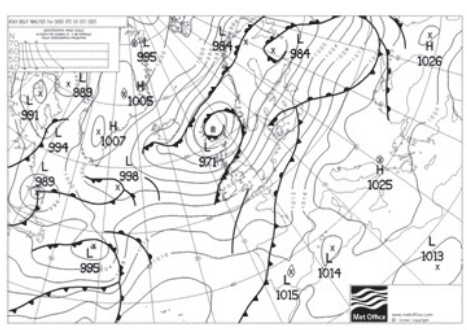

3 de Outubro

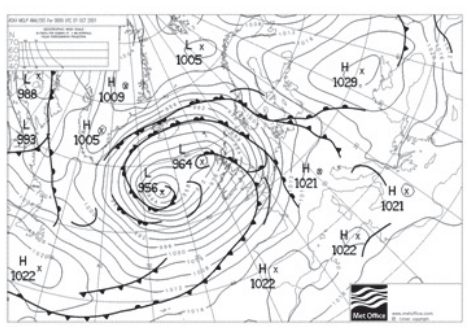

1 de Outubro

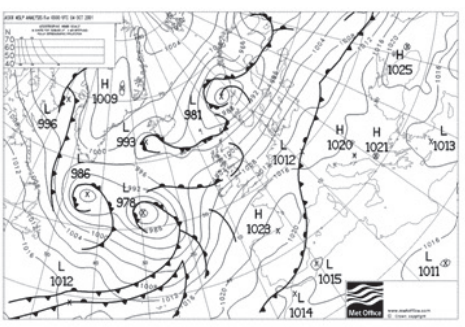

4 de Outubro

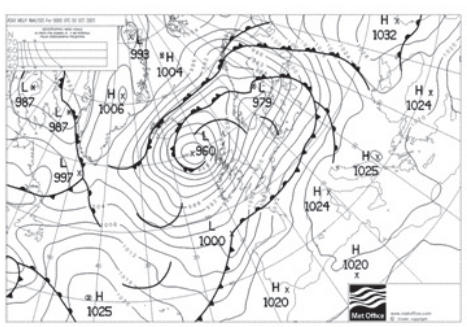

2 de Outubro

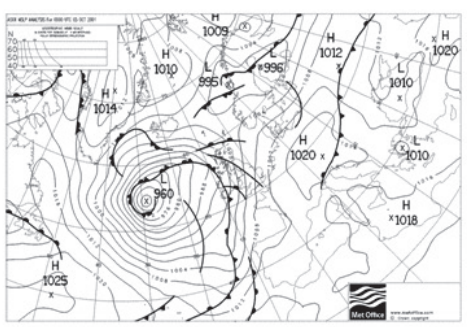

5 de Outubro

Fig. 5 - Mapas de tempo - tipo de circulação atmosférica (semana 40).

Fig. 5 - Weather maps - circulation weather types (week 40).

TABELA I - Tipos de Circulação Atmosférica, semana 39 e 40.

TABLE I - Circulation weather types, weeks; 39 and 40.

\begin{tabular}{|c|c|c|c|c|c|c|c|}
\hline Semana & Domingo & Segunda & Terça & Quarta & Quinta & Sexta \\
\hline 39 & N & N & W & SW & SW & SW \\
\hline 40 & SW & SW & SW & W & SW & SW \\
\hline
\end{tabular}




\section{Conclusão}

Os resultados obtidos mostraram inequivocamente que é possível conhecer o risco da agudização de asma e consequente aumento de acorrências hospitalares quando se analisam mapas de tempo e se investiga o tipo de circulação da atmosfera, para uma atmosfera considerada fria.

O estudo mostrou como parâmetros termohigrométricos estão ligados ao risco de acorrências hospitalares.

A análise de mapas de tempo permitiu identificar que o tipo de circulação atmosférica SW com presença de frente fria parece favorecer o risco de agudização da doença.

Os dados obtidos e a interpretação física do risco associado a agudização de asma, poderá ser uma excelente contribuição para o planeamento de serviço das urgências hospitalares, a partir do conhecimento da previsão do estado do tempo, com base nos mapas de tempo. Por exemplo, Cuba é um país em que a meteorologia tem um link com a saúde pública, ou seja, sempre que a previsão do estado do tempo indica aproximação e entrada de frente fria, o serviço de proteção civil através dos meios de comunicação alerta a população vulnerável ou de risco para se manterem resguardados em casa contra uma atmosfera considerada fria.

Por último, o ser humano terá de se adaptar às alterações climáticas; naturalmente haverá muitos problemas de natureza social e económica ligados à mudança de ecossistemas. Trabalho colaborativo deverá ser desenvolvido pela meteorologia e medicina de modo a serem encontradas as estratégias de intervenção e prevenção mais adequadas e apoiar estudos de planeamento da saúde pública.

\section{Agradecimentos}

Este trabalho é financiado pela FCT/MEC através de fundos nacionais (PIDDAC) e cofinanciado pelo FEDER através do COMPETE - Programa Operacional Fatores de Competitividade no âmbito do projeto PEst-C/CED/ UI0194/2013.

\section{Bibliografia}

Almeida, M. C. A. F. (2013). Diagnóstico de doenças obstrutivas através do registo de espirometria. Tese de mestrado em Materiais e Dispositivos Biomédicos. Publicadana Universidade deAveiro.

Almeida, C., Talaia, M. e Saraiva,A. (2012). A espirometria como teste de diagnóstico de robustez Física - Estudo de caso de uma populacão. Livro de resumos VII Encontro Nacional de Riscos e I Fórum sobre Riscos e Segurança ISCIA. Editor FEDRAVE / Mare Liberum. (pp. 56). ISCIA Aveiro. 19-20 de Abril
Boutin-Forzado, S., Adel, N., Gratecos, L., Jullian, H., Garnier, J.M., Ramadour, M., Lanteaume, A. Hamon, M., Lafay, V. and Charpin, D. (2004). Visits to the emergency room for asthma attacks and short-term variations in air pollution- A case-crossover study, Respiration, 71(2), p. 134-137.

Bush, R. K. and Prochnau, J. J. (2004). Alternariainduced asthma, Journal of Allergy and Clinical Immunology, 113(2), p. 227-234.

Cairus, H. F. e Ribeiro, W. A. (2006). Textos hipocráticos: o doente, o médico e a doença. Cad. Saúde Pública, Rio de Janeiro, 22(1), p. 233-235.

Carvalho, R. A. C. (1999). A influência das condições meteorológicas e do clima sobre doenças humanas. Condições meteorológicas, Vol(l), p. 23-45.

Decreto-Lei n. ${ }^{\circ} 79 / 2006$. O Regulamento dos sistemas energéticos de climatização em edifícios. Diário da República. I Série A, N. 67, 4 de Abril, p. 2416-2468.

Derrick, E. H. (1965). The seasonal variation in asthma in Brisbane: Its relation to temperature and humidity, International Journal Biometeorol., 9, p. 239-253.

Fanger, P. (1972). Thermal comfort (2 ed.). New York: McGraw-Hill.

Gehring, U., Heinrich, J., Jacob, B., Richter, K., Fahlbusch, B., Schelenvoigt, G., Bischof, W. and Wichmann, H.E. (2001). Respiratory symptoms in relation to indoor exposure to mite and cat allergens and endotoxins. European Respiratory Journal, 18(3), p. 555-563.

Girsh, L. S., Shubin, E., Dick C. and Schulaner F. A. (1967). A Study on the Epidemiology of Asthma in Children in Philadelphia. J. Allergy, 39, p. 347-357.

GOLD REPORT (2015). Global Initiative for Chronic Obstructive Lung Disease. Global Strategy for the Diagnosis, management and Prevention of Chronic Obstructive Pulmonary Disease (update 2015). Global Initiative for Chronic Obstructive Lung Disease, Inc. http://www. goldcopd.org/uploads/users / files/GOLD Report_2015.pdf.

Jamason, P. F., Kalkstein, L.S. e Gergen, P.J. (1997). A synoptic evaluation of asthma hospital admissions in New York City, American Journal of respiratory and critical care medicine, 156(6), p. 1781-1788.

Júlio Diniz (2005). Da importância dos estudos meteorológicos para a medicina e especialmente de suas aplicações ao ramo operatório. Fac-símile da tese apresentada na Escola Médico-Cirúrgica do Porto em 1861 por Joaquim Guilherme Gomes Coelho. Editora UP. Número da Edição $1^{\text {a }}$, Múltiplos, 1 , pp. 84

Lumdgren, F. L. C., Cabral, M. M. Climaco, D. C. S, Macedo, L. G., Coelho, M. A. L. e Dias, A. L. P .L. A. (2006). Determinação da eficiência do VEF6 como substituto da CVF na triagem diagnóstica da doença pulmonar obstrutiva crónica através da comparação entre as relações VEF1 / CVF e VEF1/ VEF6. Journal Brasilian Pneumology, 33(2), p. 148-151.

Murray, C. J. e Lopez, A. D. (1997). Alternative projections of mortality and disability by cause 1990-2020: Global Burden of Disease Study. Lancet, 349, p. $1498-1504$. 
Oga, T., Nishimura, K., Tsukino, M., Sato, S. e Hajiro, T. (2003). Analysis of the factors related to mortality in chronic obstructive pulmonary disease. Am J. Respir Crit Care Med., 167(4), p. 544-549.

Piccolo, M. C., Perillo, G. M., Ramon, C. G. and DiDio, V. (1988). Outbreaks of asthma attacks and meteorological parameters in Bahia Blanka, Argentina, Ann. Allergy, 60, p. 107-110.

Plácido, J. L. (2004). A asma a nível nacional e mundial: perspectivas actuais e tendências de evolução. http: / / www.publisaude.com/rpcgPdfs/2004/ SetOut04/n5v20583.pdf.

Portaria $n^{\circ} 702 / 80$ de 22 de Setembro. O regulamento geral de segurança e higiene do trabalho nos estabelecimentos industriais. Ministérios do Trabalho, dos Assuntos Sociais, da Agricultura e Pescas e da Indústria e Energia.

Sousa, J. C. A. M. e Talaia, M. A. R. (2005). A Gripe e a Agudização de Asma - Caso de uma Atmosfera Fria, Actas da FISICUM 2005 - FÍSICA PARA O SÉC. XXI, Edição da Sociedade Portuguesa de Física - Norte, Física Médica e Biofísica, FMB02, Porto, de 1 a 3 de Dezembro, p. 175-176.

Talaia, M. A. R. e Sousa J. C. A. M. (2004). A Saúde Pública, o Meio Ambiente e o Desenvolvimento. In: Conferência Internacional "Educação para o Desenvolvimento Sustentável - Preparação da Década das Nações Unidas (CIEDS 2004)", Departamento de Metodologias da Educacão, Instituto de Educação e Psicologia, Universidade do Minho. Braga, 19 e 22 de Maio, p. 133-134.
Talaia, M. A. R., Sousa, J. C. A. M. e Saraiva, M. A. C. (2007). Saúde e Ambiente: Como se podem relacionar na agudização de doença respiratória. Proceedings of $9^{a}$ Conferência Nacional do Ambiente, Um Futuro Sustentável - Ambiente, Sociedade e Desenvolvimento. Vol. 1, P. 154-160.

Trigo, R. M. e Câmara, C. C. (2000). Circulation weather types and their Influence on the precipitation regime in Portugal. International Journal of Climatology, 20, p. 1559-1581.

Tromp, S. W. (1980). Biometeorology: the impact of the weather and climate on humans and their environment (animals and plants): Heyden.

Suzuki, S., Kamakura, T., Tadokoto, T., Takeuchi, T., Yukiyama, Y. and Miyamoto, T. (1988). Correlation Between the Atmospheric Conditions and the Asthmatic Symptom. Int. J. Biometeorol., 32, p. 129-133.

Van, W. H. (2004). Dust mites living in human lungs - the cause of asthma? Medical Hypotheses, 63(2), p. 193-197.

Weisel, C. P., Cody, R. P., Georgopoulos, P. G., Purushothaman, V., Weiss, S. H., Bielory, L., Gregory, P. and Stern, A. H. (2002). Concepts in developing healthbased indicators for ozone. International Archives of ocucupational and Environmental health, 75(6), p. 415-422. 

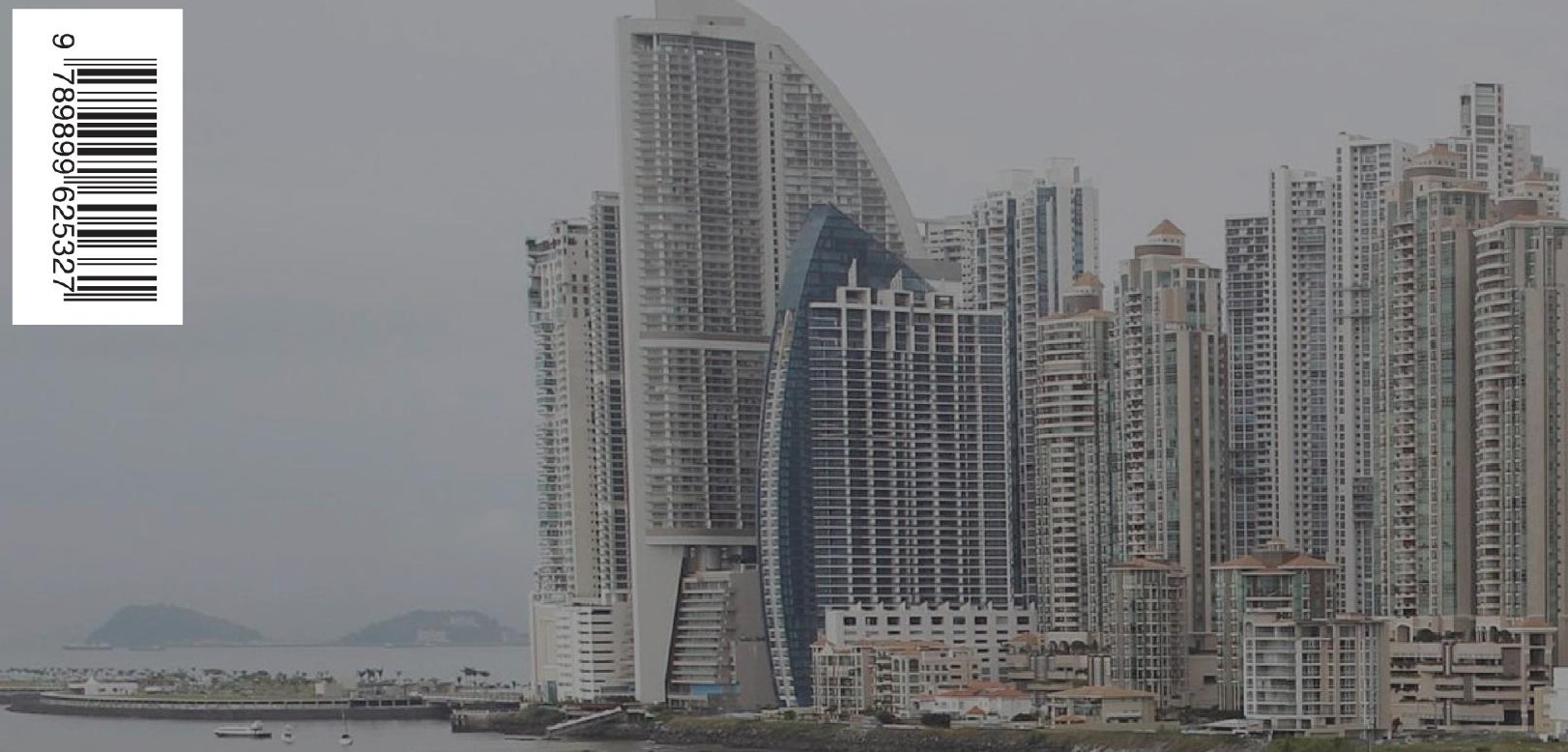

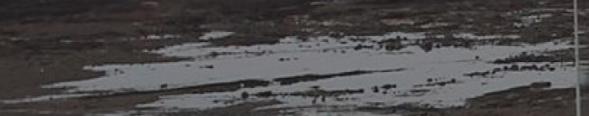

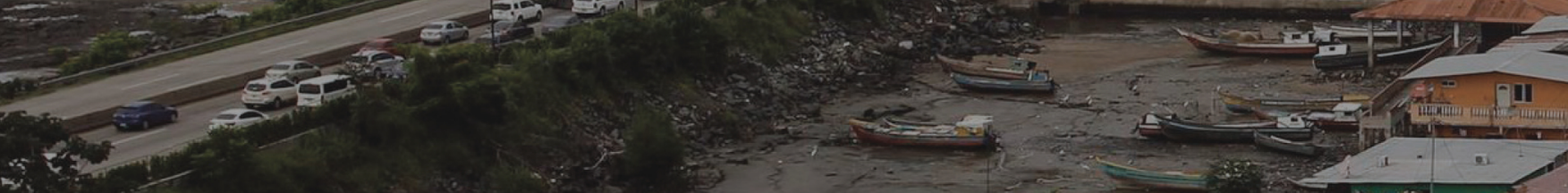

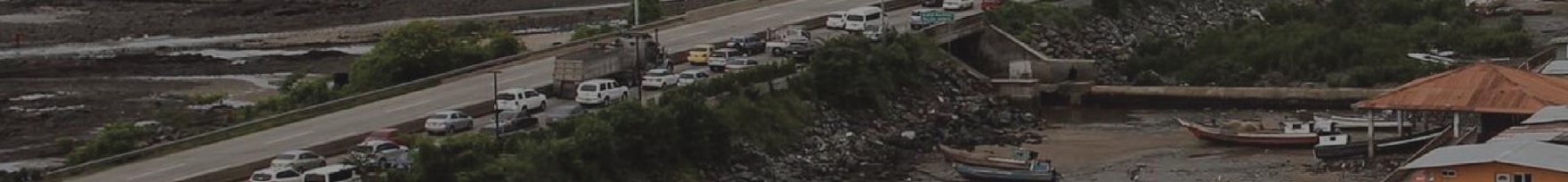
inse- $n=$ Riscos I I I

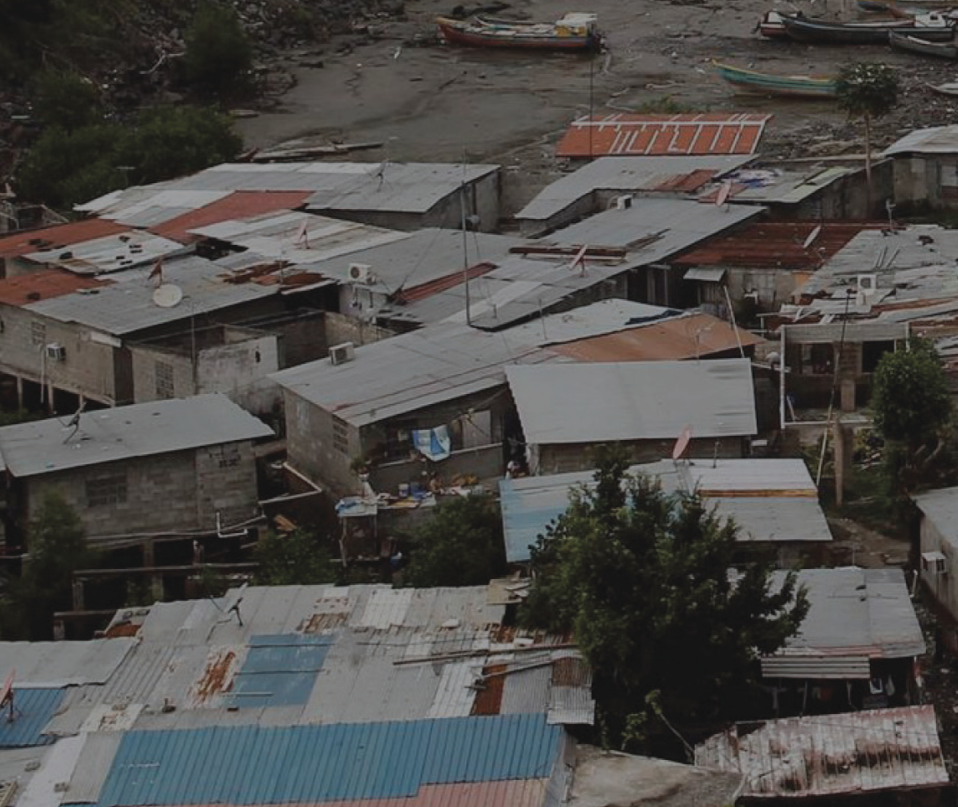

